\section{§9. Methane Decomposition Reaction on ZrNi Alloy}

Kawano, T., Sakuma, Y.,

Ohta, M. (Niigata University),

Kabutomori, T. (The Japan Steel Works Ltd.),

Shibuya, M. (JGC Corporation)

A method of decomposing hydrogen compounds was developed by employing a zirconium nickel ( $\mathrm{ZrNi}$ ) alloy. This method enables all tritium compounds ( $\mathrm{HTO}, \mathrm{CH}_{3} \mathrm{~T}$, $\mathrm{C}_{2} \mathrm{H}_{5} \mathrm{~T}$, etc.) in an exhaust gas to be decomposed into their respective elements, and the tritium itself to be removed in the form of hydrogen gas (HT). The method was developed through a series of experiments using methane. To ascertain the mechanism of methane decomposition on a $\mathrm{ZrNi}$ alloy, alloy samples were examined based on X-ray diffraction spectra before, during, and after the experiments (Fig.1.).

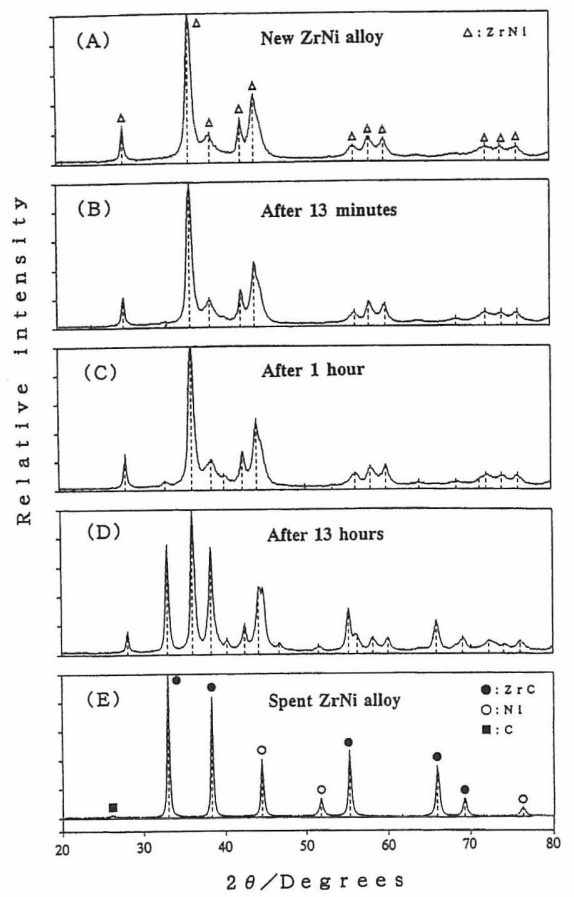

Fig. 1. Typical X-ray diffraction spectra.

As a result, the chemical reaction equation (1) explaining methane decomposition on a $\mathrm{ZrNi}$ alloy was proposed and discussed.

$$
\begin{aligned}
& \mathrm{ZrNi}+(\mathbf{1}+\alpha) \mathrm{CH}_{4} \rightarrow \\
& \mathrm{ZrC}+\mathrm{Ni}+\alpha \mathrm{C}+2(1+\alpha) \mathrm{H}_{2} .
\end{aligned}
$$

The equation (1) shows that one molecule of $\mathrm{ZrNi}$ can decompose $(1+\alpha)$ molecules of methane and generate $2(1+$ $\alpha$ ) molecules of hydrogen. The value of $\alpha$ can be determined by using the weight of the $\mathrm{ZrNi}$ used and the decomposed methane volume derived from the experiment.
The $\alpha$-value thus obtained was bigger when a larger decomposed methane volume was obtained. Therefore, if the decomposition condition is considered to be better when the total methane volume decomposed is larger, the $\alpha$-value can be used as an index to identify a good condition or not.

Under a practical good condition for us, though, the decomposition rate of more than a certain value should continue for a sufficient time length, and a larger volume of methane should be decomposed within the limited time span. It is important that a larger methane volume decomposes in a limited time span to maintain a good decomposition rate. However, the $\alpha$-value is derived based on the entire time range and is not directly connected with the limited time span. So we have to derive and examine the $\alpha$-value directly related to the limited time span. However, it is impossible to determine the $\alpha$-value of the limited time span because the weight of $\mathrm{ZrNi}$ consumed within that time cannot be determined from a practical decomposition experiment. Thus, we investigated whether the $\alpha$-value based on the entire time range can be used to evaluate the practical good decomposition condition in which a large volume of methane is decomposed within the limited time span. In the actual investigation, different decomposition rates of $10,20,30,50$, and $70 \%$ were studied and the methane volume decomposed in a partial range limited by the rates was examined. The relationship between the methane volume thus obtained and the value of $\alpha$ derived from the entire range was investigated.

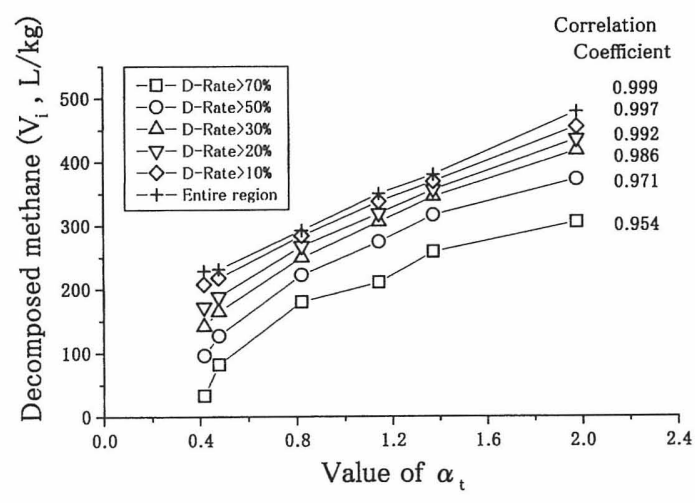

Fig. 2. Correlation between value of $\alpha_{t}$ and decomposed methane volume of $V_{i}$.

A result is shown in Fig.2. In Fig.2, the value of $\alpha$ was found to be strongly correlated to the methane volume decomposed within the limited time span. We thus conclude that the $\alpha$-value based on the entire time range can be used as an index to estimate whether a decomposition condition is better or not from the view point of actual processing work. 